\title{
RPC monitoring tools in the CMS experiment
}

\section{Umberto Berzano for the CMS Collaboration}

CERN (Switzerland) and Università degli Studi di Pavia (Italy)

E-mail: Umberto.Berzano@cern.ch

Resistive Plate Chambers have been used in the CMS experiment to build up a dedicated muon detector system which covers the whole geometric acceptance up to a pseudorapidity of 1.6 with a total of 480 chambers in the barrel and 432 in the endcaps. In order to guarantee the quality of the data collected and to monitor online the detector performance, a set of common tools has been developed in CMS and they are in particular heavily used in the RPC system: the Web Based Monitoring tools and the Data Quality Monitor system. The first ones are a set of templates available on the web which allow the user to check the hardware performance during data taking with distributions and plots of all the parameters. The Data Quality Monitoring service is composed by a set of algorithms which can work both during data taking and offline, running on stored data, to check the quality of reconstructed physical variables. Both these monitoring systems will be described here with a particular emphasys on their structure, functionalities and performance. 


\section{Introduction}

In the Compact Muon Solenoid (CMS) [1] experiment the Resistive Plate Chambers (RPC) [2] represent a complex and massive subdetector system with a total of 912 double-gap chambers installed in the CMS magnet return yoke. The detector status, the performance and the data certification are crucial aspects for the correct apparatus operation. All these critical tasks are carried out by 2 different software frameworks: the Web Based Monitoring (WBM) tools and the Data Quality Monitoring (DQM) system. They are both used by the shifters and by the detector experts during the online data-taking phase and in the offline data certification.

\section{The Web Based Monitoring tools}

A big experiment like CMS with millions of sensor channels, infrastructure features and complex trigger and DAQ systems, requires an accurate monitoring system. The goal of CMS WBM [3] is to provide a suite of tools which is secure and flexible, easy to use and to maintain, and is accessible worldwide by the collaboration. A simplified view of the WBM system architecture is displayed in Figure 1.

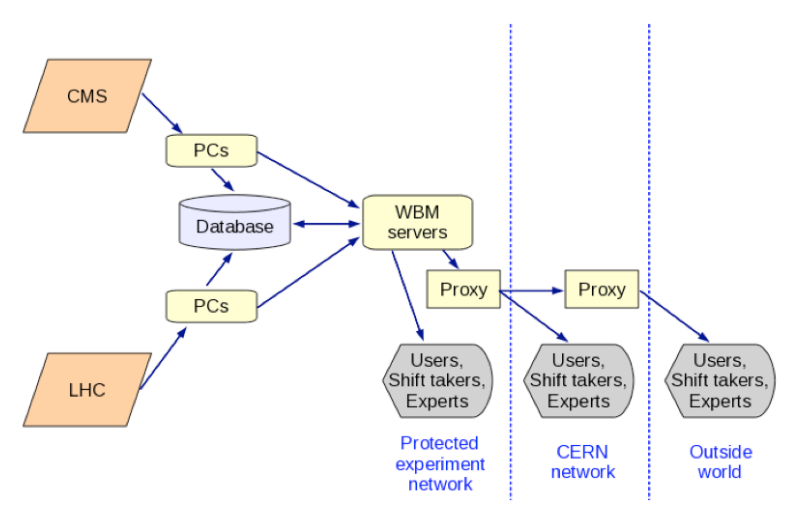

Figure 1: Overview of the systems for the WBM services.

The RPC WBM is part of the CMS WBM service running on the WBM production servers. It is a set of JAVA servlets which use SQL (Structured Query Language) to communicate with the CMS online database and which run ROOT [4] macros to produce plots and informations on the hardware performance. All the main parameters of the system can be monitored through WBM - high voltage, current, temperature, gas flow, noise - as well as the environmental variables. These features allow both the expert user and the shifter to have a clear idea of the apparatus performance during a run or for a longer period of time. 
As an example of the potentiality of the service, the RPC current monitoring is considered. The chamber currents, averaged on wheels and disks, are plotted vs. time in Figure 2. It is worth to observe that the RPC current depends on the shape of the LHC proton beams intensity till the dump of the beam visible in the right part of each plot.
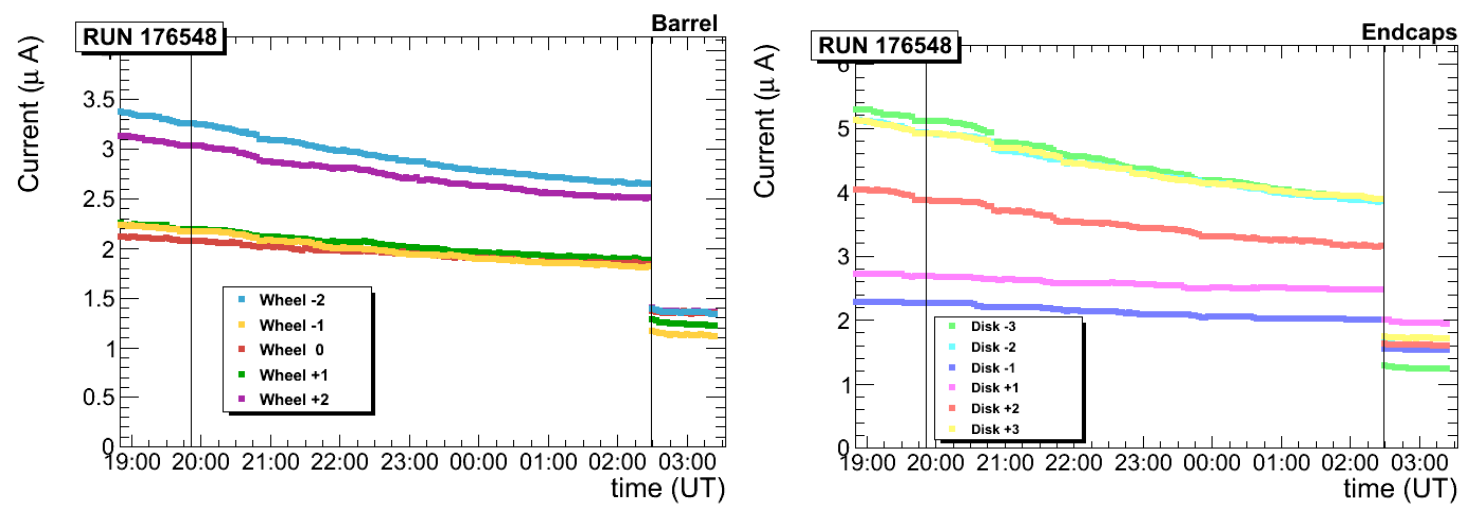

Figure 2: History plots of the chamber current during a run averaged on barrel wheels (left) and on endcaps disks (right). The start and stop of the run are indicated by two vertical black lines.

The luminosity dependance of the RPC currents can be investigated further by using WBM tools. For this purpose, four runs with increasing LHC luminosity are chosen, analyzed and the resulting histograms are compared. Figure 3 shows the maximum current reached in a run by the endcap chambers. The systematic shift of the distributions with the increasing of the luminosity of the beams is quite evident (left plot). The trend of the current increase depends on the disk (right plot). Indeed, it is caused by the background radiation level which depends on the position of the disks relative to the CMS interaction point.
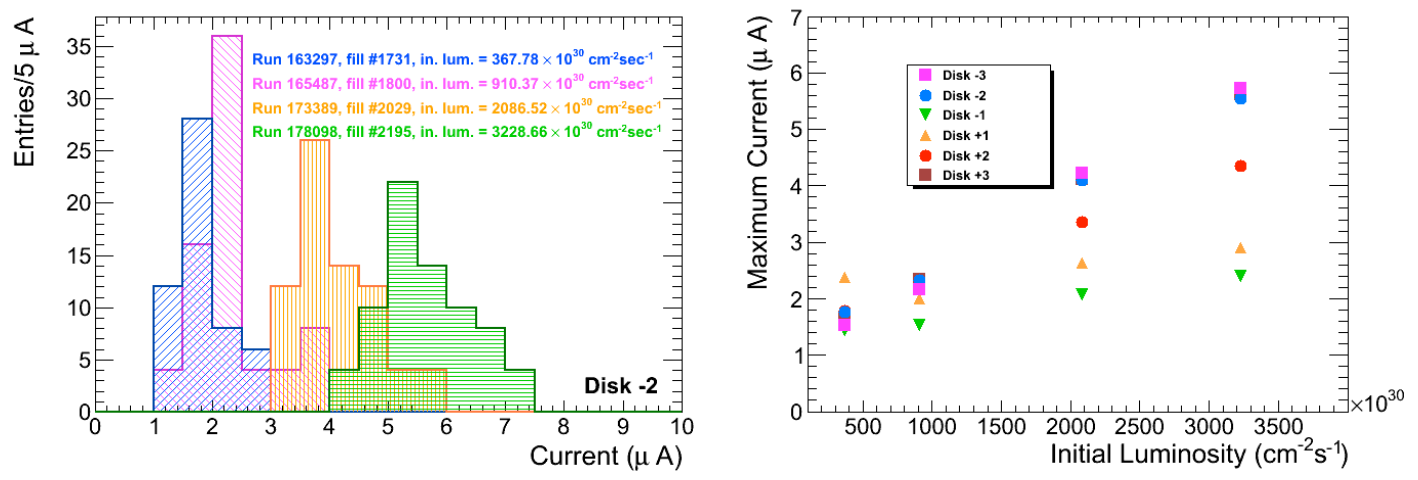

Figure 3: Maximum current distributions for different beam luminosity in an endcap disk (left). The mean value of these distributions is plotted for all the endcap disks to show the dependance of the current on the luminosity (right). 
The shifter or the detector expert also need to monitor the single chambers behaviour during a run and so the WBM service provides the maximum and the average currents of all the chamber in the RPC system. Figure 4 shows a map of the average current of all the chambers in one of the barrel wheels during a run. From this plot the shifter can identify unambigously if a chamber is running at a higher current than expected and in that case call the experts for a deeper analysis.

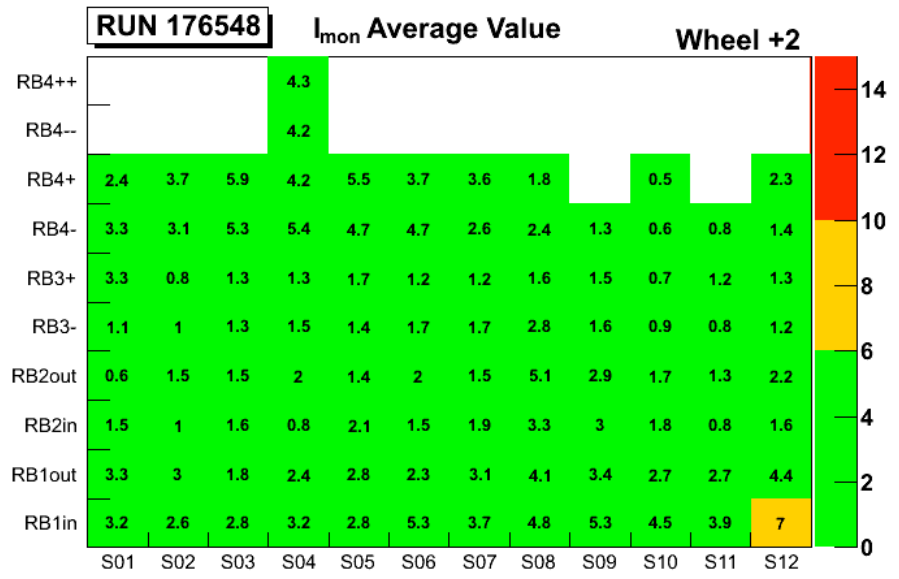

Figure 4: Map of the average chamber currents in a wheel for a given run. The normal working current has a maximum set at $6 \mu \mathrm{A}$. If the average current of a chamber is $>10 \mu \mathrm{A}$ an expert must check it.

Together with other detector variables, the current monitoring is the first step of the data certification the shifter must provide soon after the data are available. After having verified the detector hardware, the data integrity and quality can be easily checked with the Data Quality Monitoring service.

\section{The Data Quality Monitoring tools}

The CMS DQM system [5] provides a set of tools for the creation and storage of histograms and other elements related to the detector performance and the collected data quality. It is intended to be used both online, during data taking, and offline, during the event reconstruction and data validation.

The online DQM is an integral part of the event data processing and it can monitor the detector, trigger and DAQ hardware statuses in real time. The output consists of histograms, alarm states and quality test results for a total of about $10^{3}$ items. All this information is stored in ROOT files, intended for expert usage, and is uploaded to the central graphical user interface (GUI) accessible from the web. As for WBM, the web-based nature of the service permits users 
all over the world to access the data and to check results without having to install any additional software. A screen shot of the DQM GUI is reported in Figure 5.

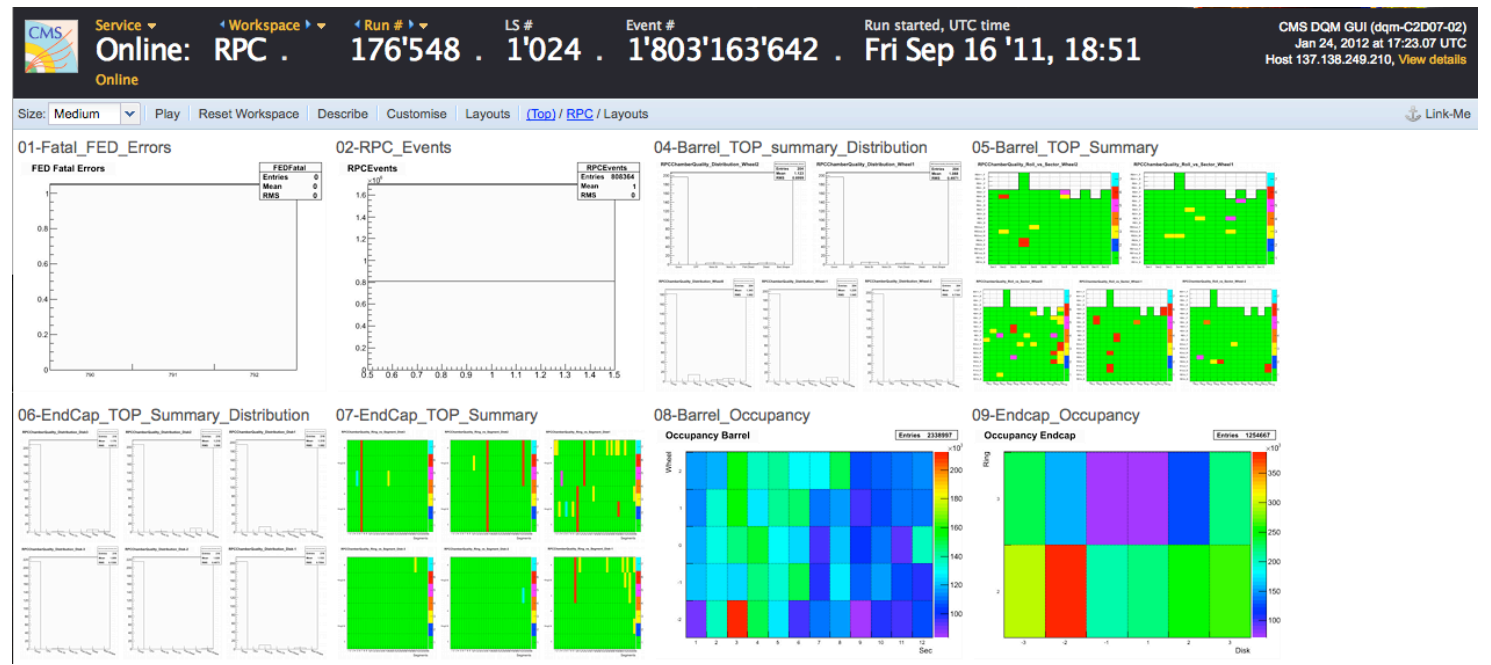

Figure 5: The DQM GUI.

The offline DQM is used to certify the quality of recorded data and the detector performance. About $10^{4}$ histograms are produced by this system relating all the main relevant variables for the performance of RPCs such as chamber occupancy, strip multiplicity, number of clusters per chamber, bunch crossing number, chamber efficiency and noise level. The information contained in these histograms is crucial for the manual run certification performed by the shifter who is asked to look at a defined set of summary histograms and to take a decision about the data quality.

The end of the certification task is a list of "good" and "bad" runs, which is eventually copied to the offline condition database.

\section{References}

[1] CMS Collaboration, "The Compact Muon Solenoid Technical Proposal", CERN/LHCC-94-38 (1994).

[2] M. Abbrescia et al., "The RPC system for the CMS experiment at the LHC", Nucl. Instrum. Methods A 508 (2003) 137-141.

[3] A. Soha et al., "Web Based Monitoring in the CMS Experiment at CERN" Proc. CHEP10, Computing in High Energy and Nuclear Physics (Taipei, Taiwan), 2010.

[4] http://root.cern.ch/

[5] L. Tuura et al., "CMS data quality monitoring: systems and experiences" Proc. CHEP09, Computing in High Energy and Nuclear Physics (Prague, Czech Republic), 2009. 